\title{
¿Por qué directores y tesistas deciden discontinuar la relación pedagógica?
}

Why do Supervisors and Thesis Students Decide to Discontinue the Pedagogical Relationship?

Artículo de investigación| Research article

Fecha de recepción: 23 de noviembre de 2019 Fecha de aceptación: 12 de noviembre de 2020

Fecha de disponibilidad en línea: Agosto 2021

doi: 10.11144/Javeriana.m14.pqdt

Lorena Fernández-Fastuca lorena_fernandez@uca.edu.ar Pontificia Universidad Católica Argentina, Argentina D ORCID: https://orcid.org/0000-0002-8033-6158

Para citar este artículo | To cite this article Fernández-Fastuca, L. (2021). ¿Por qué directores y tesistas deciden discontinuar la relación pedagógica? magis, Revista Internacional de Investigación en Educación, 14, 1-26. doi: 10.11144/Javeriana.m14.pqdt 


\title{
Resumen
}

La dirección de tesis es un pilar de la formación de posgrado. Por ello, se propone analizar la relación director-tesista indagando las razones que pueden llevar a su discontinuidad desde la perspectiva de ambos. Se realizó un estudio cuantitativo descriptivo, con base en una encuesta diseñada ad hoc, que se aplicó a una muestra no representativa de 1607 directores y tesistas de universidades argentinas. El 58\% de los directores ha discontinuado este vínculo. Algunas razones son: insuficiente dedicación del tesista e insuficiente seguimiento de las indicaciones. Para los tesistas el motivo central es la falta de dedicación del director.

\section{Palabras clave}

Posgrado; relación profesor-alumno; tesis; tutoría

\begin{abstract}
Thesis supervision is a pillar of postgraduate education. We analyzed the supervisor-thesis relationship investigating the reasons that may lead to its discontinuity from the perspective of both parties. A descriptive quantitative study was made, based on an ad hoc designed survey, which was applied to a non-representative sample of 1607 thesis supervisors and thesis students from universities in Argentina. Results indicated that $58 \%$ of thesis supervisors discontinued their relationship. Some reasons they provided were: Insufficient dedication of the student and insufficient following of the recommendations. For thesis students, the central reason was the supervisor's lack of support.
\end{abstract}

\section{Keywords}

Postgraduate courses; student teacher relationship; theses; mentoring 
Descripción del artículo | Article description

Artículo de investigación derivado del proyecto de

investigación de la beca posdoctoral Los problemas

en la dirección de tesis. Motivos que llevan a

descontinuar la relación y repercusiones para cada

uno y el programa doctoral.

\section{Introducción}

En el ámbito universitario estamos acostumbrados a los relatos sobre las vicisitudes del proceso de realizar una tesis $y$, en ellos, el rol preponderante que suele tomar la figura del director (ya sea porque ha sido una guía y maestro, ya sea porque la tesis se ha realizado a pesar de sus intervenciones o ausencias). La familiaridad de esta figura no se corresponde con conocimiento pedagógico-didáctico profundo sobre ella. En efecto, hace pocas décadas que se están produciendo investigaciones sobre ella; el interés se originó principalmente como consecuencia de los estudios que analizan las tasas de abandono de los posgrados (Bartolini, 2017; Bowen \& Rudenstine, 1992; De Miguel et al., 2004; Wainerman, 2017) y los factores asociados a estas, entre los que se identifica como la principal causa la dirección de tesis (Carlino, 2005; Ehrenberg et al., 2010; Ferrer de Valero, 2001; Gardner, 2009; entre otros). Estos últimos utilizan diseños basados en encuestas, entrevistas o ambos con el fin de estudiar dichos factores y concluyen que la calidad de la dirección de tesis es uno de los principales intervinientes en las posibilidades de graduación.

Así, la dirección de tesis es uno de los pilares de la formación de posgrado. En este artículo nos proponemos analizar la relación pedagógica entre director y tesista en programas de posgrado (maestría y doctorado) a partir de la indagación de las razones que pueden llevar a la disolución de dicha relación y las consecuencias que conlleva para la formación del tesista. Focalizarnos en "el lado oscuro" de la dirección de tesis nos permite alcanzar un mayor conocimiento sobre sus características y notas fundamentales.

La relevancia de esta relación pedagógica promovió nuevos estudios que abordan dimensiones variadas. Así, algunos indagan las características deseables en una relación director-tesista (Abiddin \& West, 2007; De la Cruz-Flores et al., 2006; Difabio, 2011; Fraser \& Mathews, 1999; Hasrati, 2005; Kiley, 2011; Marsh et al., 2002; Subhajyoti, 2007), los estilos de dirección posibles (Bell-Ellison \& Dedrick, 2008; Deuchar, 2008; Farji-Brener, 2007; Fernández-Fastuca, 2019; Johnson et al., 2000; Mainhard et al., 2009; Murphy et al., 2007) y unos pocos sobre los problemas en la relación 
director-tesista (Delamont \& Atkinson, 2001; Devos et al., 2016; Galetto et al., 2007; Hidalgo \& Passarella, 2009; Löfström \& Pyhältö, 2015). Entre estos estudios, Delamont \& Atkinson (2001) advierten sobre las expectativas que desarrolla el tesista acerca del rol del director para responder a las tensiones que genera la incertidumbre propia de las distintas etapas del proceso doctoral. Hidalgo \& Passarella (2009) se centran en las diferencias en las expectativas sobre la relación que tiene cada parte, mientras que Devos et al. (2016) amplían la mirada al abordar los distintos tipos de desajustes que puede atravesar la dirección de tesis (en las expectativas respecto del proyecto de investigación; entre el estilo del director y las expectativas del tesista, y con relación a lo que constituye una buena investigación para cada uno). Por su parte, Löfström \& Pyhältö (2015) se adentran en la ética en la dirección de tesis y en los problemas que pueden originarse al incurrir en faltas a ella. Finalmente, Galetto et al. (2007) ponen el acento en las características del tesista y cómo inciden en el buen desarrollo del proceso formativo. En síntesis, esta línea de investigación generalmente analiza las tensiones que se producen, sin profundizar en sus consecuencias ni considerar la problemática pedagógica subyacente.

La discontinuidad de una relación director-tesista tiene repercusiones para cada una de las partes de la relación: ¿Qué tan frecuente es esta situación en el ámbito académico?, ¿cuáles perfiles de investigadores están más expuestos a la discontinuidad de las direcciones?, ¿existen diferencias entre los grupos disciplinares?, ¿qué motivos llevan a la disolución de una relación de dirección de tesis?, ¿qué posibilidades de continuidad existen para el tesista? Aquí buscamos comprender en mayor profundidad la dirección de tesis como fenómeno formativo y las particularidades que encierra en cuanto al tipo de relación educativa privativa del nivel universitario y, especialmente, de los estudios de posgrado.

\section{Referentes conceptuales}

La dirección de tesis es uno de los dispositivos pedagógicos centrales de las carreras que requieren la realización de una tesis. Si bien su dimensión formativa ha sido pasada por alto y se ha apoyado principalmente en el tradicional modelo maestro-aprendiz (según el cual se aprende a investigar a partir de la observación de la actividad del maestro, quien transmite el método y la teoría), posturas actuales resaltan la necesidad de explicitar los conocimientos y comunicarlos a los aprendices, así como la importancia de la función tutorial del director en la formación (De la Cruz-Flores et al., 2006; Halse, 2011; Manathunga, 2007). Este cambio de concepción se basa en el reconocimiento de que el tesista no es completamente 
autónomo en cuanto a su proyecto de investigación refiere (Johnson et al., 2000; Manathunga \& Goozée, 2007); en parte debido a las diferencias que existen entre la asistencia a cursos y la producción de la tesis (Cassuto, 2010; Diezmann, 2005; Moreno-Bayardo, 2003; entre otros). El paso de la asistencia a cursos a la etapa de tesis implica dejar de contar con un docente que organice las tareas de aprendizaje (los contenidos, la bibliografía, el cronograma, las actividades por realizar) y, además, posicionarse como "productor de conocimientos" (Lovitts, 2005). De ahí, la necesidad de guía y orientación, y la concepción de la parcial autonomía del tesista. Así, la dirección de tesis es una relación pedagógica "en la cual el director tiene una intención explícita de promover, a partir del desarrollo de actividades, el aprendizaje de determinados conocimientos o habilidades por el tesista" (Fernández-Fastuca \& Wainerman, 2015, p. 160). Entre director y tesista se produce una alianza de aprendizaje según la cual el director se responsabiliza por la formación del segundo (Halse \& Malfroy, 2010).

Esta relación es, sobre todo, un vínculo entre dos personas. Toda relación pedagógica se basa en el reconocimiento mutuo, y en que el estudiante se identifique con el formador y quiera emularlo en algún aspecto (Jackson, 2015). Parte de ese reconocimiento también es el establecimiento de una relación que permita al otro alcanzar sus objetivos de formación. Para el caso de la dirección, De la Cruz-Flores et al. (2006) identifican como una de las funciones principales del director de tesis el apoyo psicosocial, esto es, brindar las condiciones sociales y emocionales necesarias para que el tesista pueda realizar su estudio.

En esta misma línea, la investigación es una actividad creativa que involucra a la totalidad del yo (Wainerman, 2011). Es decir, es una actividad que para el tesista siempre involucra su subjetividad; no solo porque es un acto creativo (de producción de conocimiento científico), sino también porque implica la consecución de un grado académico y, por ende, el logro de objetivos propios. La investigación realizada por Hidalgo \& Passarella (2009, p. 74) ayuda a evidenciar este involucramiento del tesista, al señalar que "la complejidad de la elaboración de una tesis, tanto cognoscitiva como del orden de la escritura, parece siempre desbordar los límites de la guía que los tesistas obtienen de sus directores".

Desde el otro lado de la relación, Gilmore et al. (2016) llegan a la conclusión de que los directores que entrevistaron no lograban ver cómo su accionar afectaba la motivación y el desempeño del tesista. O sea, parece tratarse de una relación pedagógica en la que cada parte tiene expectativas distintas sobre lo que esta debe ser, el rol propio y el rol de la otra parte del binomio. Åkerlind \& McAlpline (2017) señalan que el acuerdo entre director y tesista en el propósito con el que se realiza el doctorado es esencial para 
la relación de dirección. Además, identifican dos dimensiones en estos objetivos, que son: qué se está intentando lograr y por qué lo están intentando. Y señalan que:

la variación que existe en ambos aspectos debe tenerse en cuenta tanto en la educación doctoral como en el desarrollo profesional del supervisor. A menos que dicha variación en los propósitos se haga explícita, crea una impresión implícita de una mayor uniformidad entre estudiantes y supervisores de lo que realmente es el caso, aumentando las posibilidades de falta de comunicación y malentendido. (2017, p. 1696)

Asimismo, como en toda actividad formativa, los directores pueden asumir distintos estilos formativos (Fernández-Fastuca, 2019); esta diversidad de estilos puede llevar a problemas en la relación pedagógica. Por ejemplo, en ocasiones el rol activo del director genera tensiones entre su intervención y la autonomía del tesista (Delamont et al., 1998). Paralelamente, Deuchar (2008) ha puesto en evidencia que los tesistas suelen necesitar distintos tipos de orientación, lo que requeriría que los directores puedan modificar sus estrategias de un tesista a otro. Además, un mismo tesista puede tener necesidades distintas a lo largo del proceso de investigación y establece el vínculo con el director también de modo diferente. Como señalan Bradbury-Jones et al. (2007), los momentos de mayor proximidad entre director y tesista ocurren cuando este requiere de más orientación, mientras que a partir del avance en la investigación y del crecimiento como investigador se produce un desapego progresivo.

En la Argentina, Hidalgo \& Passarella (2009) han analizado las representaciones que los tesistas manifiestan sobre el vínculo pedagógico con el director y logran identificar algunas tensiones que se desprenden de ellas. De acuerdo con las autoras, estas se diferencian entre hombres y mujeres. En el primer caso, generalmente atribuyen estas dificultades a cuestiones coyunturales, como "los múltiples compromisos del/la director/a, las condiciones precarias o de infraestructura en que llevan a cabo la tarea, dedicación parcial a la tesis o bien a rasgos negativos de la personalidad del tutor" (Hidalgo \& Passarella, 2009, p. 76). En el caso de las mujeres, es la dimensión afectiva la que se pondera a la hora de analizar las tensiones, así como la idoneidad para la tarea de dirección.

Otro foco de tensiones, acorde con Löfström \& Pyhältö (2015), son las discrepancias en la ética académica, que ocurren principalmente porque directores y tesistas tienen expectativas diferentes respecto de la dirección de tesis. Las autoras hallaron tres principios éticos importantes para directores y tesistas: evitar el daño — sin mala intención - (que se traduce en preocupación por la explotación tanto en tesistas como en directores, la 
apropiación indebida de resultados y relaciones duales), ser justo (para los tesistas refiere a experiencias de inequidad y asignación injusta de crédito por su contribución al proyecto) y ser fiel (que se traduce para los tesistas en miedo al abandono por parte de sus directores e inadecuada dirección). Contrariamente a sus hipótesis, no encontraron relevancia en los siguientes principios: respeto por la autonomía (derecho de las personas a la toma de decisiones sobre sus vidas) y beneficio para otros (lo que implica una contribución al bienestar de los demás).

Por su parte, Devos et al. (2016) identifican tres tipos de desajuste entre directores y tesistas: 1) en cuanto a las expectativas respecto del proyecto de investigación (el tema específico que desean investigar, el abordaje metodológico), 2) con relación a lo que constituye una buena investigación para cada uno y sus prioridades en la vida y 3 ) las discrepancias entre el estilo del director y las necesidades y expectativas del tesista. Un aporte interesante de este estudio es que avanza en identificar cuatro tipos de actitudes del tesista frente al desajuste:

1) Aprender a vivir con él, 2) sufrir la situación sin poder abordarlo con su director, 3) abordar el problema e intentar resolverlo de diferentes modos, o 4) ser incapaz de intentar regular el problema ya que se ha alcanzado un punto de no retorno. (2016, p. 475)

Este último punto abre un abanico de preguntas sobre cuáles son los motivos que conducen a este "no retorno". ¿Son las diferencias en la modalidad de trabajo, la incompatibilidad teórica, las discrepancias en el diseño metodológico, cuestiones personales u otros motivos?, ¿es posible realizar una lectura pedagógica de esos motivos?, ¿qué lugar ocupa el programa doctoral en estas situaciones? son algunas de las preguntas que buscamos profundizar en esta investigación.

\section{Metodología}

Este estudio cuantitativo descriptivo (Bisquerra-Alsina, 2009) constituye una "puerta de entrada" al estudio de la problemática en el contexto argentino. Nos permite indagar su alcance, las características de los directores expuestos al fenómeno, y obtener datos sobre los motivos que inciden en la disolución de la relación director-tesista.

Se realizó una encuesta dirigida a dos grupos: 1) directores de tesis y 2) tesistas, ya sean graduados o no, de programas de posgrado. La población de directores de tesis y tesistas estuvo conformada por académicos e investigadores que ocuparan (o hubieran ocupado) dichos roles. Por lo tanto, si bien se construyeron dos cuestionarios independientes, 
se distribuyeron en un mismo correo electrónico para que los individuos pudieran elegir desde cuál rol responder. ${ }^{1}$ Una vez construidos los cuestionarios se sometieron a la evaluación de dos investigadores con experiencia en este instrumento; y, una vez volcadas sus sugerencias, se realizó un estudio piloto dirigido a diez investigadores de distintas disciplinas, con quienes luego se tuvo una conversación sobre los cuestionarios. Nuevas modificaciones a los instrumentos se hicieron como resultado de dicho estudio piloto.

Con el objetivo de incluir directores y tesistas de distintas universidades del país, se efectuó una encuesta autoadministrada digitalmente y distribuida por correo electrónico, dirigida a una muestra no probabilística por conveniencia. En concreto, esta fue enviada a: investigadores cuyas direcciones de correo electrónico estuvieran publicadas 1) en el área investigación de los sitios web de las 118 universidades argentinas (de gestión estatal y privada) existentes en 2018 y 2) en sitios web de Institutos de investigación argentinos; también a 3) programas doctorales y de maestría de todas las universidades del país (solicitando que se enviara la encuesta a graduados y alumnos) y 4) a contactos personales dentro del ámbito académico. Una limitación de la muestra fue que el contacto con los tesistas estuvo dirigido a aquellos que luego continuaron su trayecto profesional académico o "mediado" por los programas de posgrado. Ello pudo haber limitado el acceso a quienes han abandonado sus estudios. Sin embargo, como nuestro objetivo era indagar los problemas que llevan a la disolución de la relación directortesista, este método de selección nos permitió potenciar las posibilidades de llegar a aquellos que discontinuaron la relación con el director de tesis, pero que no necesariamente abandonaron el posgrado.

La encuesta se remitió a un total de 11841 direcciones de correo y se recibieron 1607 respuestas (865 de los directores y 742 de tesistas). Los cuestionarios se distribuyeron entre julio y noviembre de 2018 y la encuesta estuvo habilitada para recibir respuestas hasta el 30 de diciembre del mismo año. Otra de las limitaciones del tipo de administración y muestreo fue que tuvimos acceso solo a quienes estuvieron interesados en la problemática (quizás por haberla vivenciado) y en participar de la investigación (Bisquerra-Alsina, 2009).

En los dos cuestionarios, las variables fueron prácticamente las mismas, aunque se tradujeron en preguntas distintas; a saber: perfil del encuestado (edad, disciplina de formación, lugar de trabajo, mayor título alcanzado, antecedentes profesionales), experiencia en la dirección de tesis, experiencia

1 Dado el carácter anónimo de la encuesta, no es posible saber quiénes lo han hecho desde cada rol. 
de haber discontinuado un vínculo director-tesista, autoría de la decisión y motivos que se adjudican a la discontinuidad. Ambos cuestionarios se componen de 20 preguntas, en su mayoría cerradas. En cuanto a las principales variables del estudio, para indagar la experiencia en la dirección se pidió indicar la cantidad de tesis dirigidas y finalizadas en los distintos grados de formación (licenciatura, maestría, doctorado) y la cantidad de tesis en proceso en cada grado. En cuanto a la discontinuidad se preguntó: "Entre las tesis que ha dirigido, ¿alguna se interrumpió definitivamente o parece haberse discontinuado? (ya sea por decisión suya o del tesista)". Para luego indagar la cantidad y el grado de pertenencia de las tesis discontinuadas. Para los tesistas las preguntas sobre la discontinuidad fueron: "¿En tu experiencia como tesista dejaste alguna vez de trabajar con un director antes de terminar la tesis?", "La tesis para la cual has dejado de trabajar con un director ¿a qué grado académico pertenecía?", "La decisión de discontinuar el proceso de dirección, ¿de quién fue? (con las opciones: propia, del director, de ambos, no hubo una decisión explícita)", y "¿Cuál ha sido la consecuencia de la discontinuación de la dirección de tesis? (opciones de respuesta: abandono de la carrera, cambio de director)". Estos son algunos ejemplos de las preguntas que conformaron el cuestionario. Además, se realizaron dos preguntas abiertas: una de ellas pedía a los encuestados que relataran el motivo de discontinuidad del vínculo pedagógico, y para los tesistas también se incluía la especificación de "otros motivos" en una pregunta cerrada que ofrecía motivos predeterminados².

Finalmente, para el análisis de los resultados se realizó un análisis descriptivo univariado y bivariado, utilizando los software Excel y SPSS. Para las preguntas abiertas seguimos, como señalan Maxwell \& Miller (2012), una estrategia basada en la contigüidad (contiguity-based), que reúne tanto la categorización como los emergentes del campo. Por lo tanto, hubo categorías predefinidas con base en la revisión bibliográfica (Åkerlind \& McAlpline, 2017; Kam, 1997; Löfström \& Pyhältö, 2015; Torres-Calixto, 2011) y otras que se construyeron como emergentes.

\section{Resultados y discusión}

Comenzaremos presentando las características de la muestra que, si bien no es representativa del universo de directores y tesistas, por su conformación en términos del perfil de los respondientes, contextualiza el análisis del vínculo pedagógico entre ambos. De los 865 académicos que

2 En el presente artículo se ha optado por no presentar los resultados relativos a esa pregunta. 
respondieron la encuesta en calidad de directores de tesis, el $57 \%$ son mayores de 50 años y el $53 \%$ son mujeres.

El $88 \%$ de los directores de tesis son doctores. Respecto de la distribución entre las áreas disciplinares, los grupos con mayor representación en la encuesta son Ciencias Sociales y Humanas (32\%); Ciencias Biológicas y de la Salud (30\%); Ciencias Naturales y Exactas (23\%), y Ciencias Agrarias, de la Ingeniería y Tecnología (15\%). En este punto, la representación de las distintas áreas disciplinares se corresponde con la distribución de los investigadores del Consejo Nacional de Investigaciones Científicas y Técnicas (Conicet)³ ${ }^{3}$ a excepción de las Ciencias Sociales y Humanas, que están levemente más representadas.

En cuanto a su inserción institucional, la mayoría trabaja en una universidad o instituto de investigación del sector estatal ( $88 \%$ ) y un $8 \%$ en sus equivalentes del sector privado. Además, el $43 \%$ de los directores encuestados pertenece al Conicet, las principales categorías son independiente (33\%), principal (29\%) y adjunto (14\%). Además, sean miembros de dicho organismo científico o no ${ }^{4}$, el $81 \%$ de los directores de la muestra tienen una dedicación de la mitad de su tiempo o superior a la investigación. Esto significa que, si consideramos que la dirección de tesis es una tarea propia de la actividad académica, quienes respondieron lo hicieron desde un lugar de experiencia y pericia en la tarea. En este sentido, si atendemos a la cantidad de tesis dirigidas y finalizadas, quienes han dirigido seis o más tesis de maestría y doctorado son quienes mayor tiempo dedican a la tarea investigativa (100\% y $97 \%$, respectivamente).

Por su parte, el $62 \%$ de los tesistas encuestados tienen menos de 40 años en el momento de la encuesta. Además, el $62 \%$ son mujeres. Del total, el $81 \%$ se desempeña laboralmente en el ámbito académico. El 77\% de ellos lo hacen en investigación (exclusivamente, el 17\%), un 69\% realiza también tareas de docencia y un 16\% agrega la gestión.

En cuanto al área disciplinar (tabla 1), al igual que con los directores, las Ciencias Sociales y Humanas tienen mayor representación, con un 46\%, seguidas por las Ciencias Biológicas y de la Salud con un $29 \%$ de los respondientes. Contrario a la conformación de la muestra para los directores, en este caso las Ciencias Exactas y Naturales tienen menor porcentaje que las Ciencias Agrarias, de la Ingeniería y Tecnología.

3 Según dicho organismo, los investigadores se distribuyen del siguiente modo: Ciencias Agrarias, 23\%; Ciencias Biológicas, 30\%; Ciencias Exactas, 22\%; Ciencias Sociales y Humanas, $22 \%$, y Tecnología, $3 \%$.

4 Entre los miembros del Conicet, el 77\% dedica la mayor parte de su tiempo a la investigación, y un $21 \%$ le dedica la mitad. Entre quienes no pertenecen al Conicet, el $24 \%$ ocupa la mayor parte de su tiempo en la actividad investigativa y el $57 \%$, la mitad. 
Tabla 1

Distribución porcentual de los tesistas según área disciplinar de formación

\begin{tabular}{l|c} 
& Porcentaje \\
\hline Ciencias Agrarias, de la Ingeniería y Tecnología & $13,1 \%$ \\
\hline Ciencias Biológicas y de la Salud & $29,6 \%$ \\
\hline Ciencias Sociales y Humanas & $46,5 \%$ \\
\hline Ciencias Exactas y Naturales & $8,1 \%$ \\
\hline NS/NR & $2,7 \%$ \\
\hline Total general & $\mathbf{1 0 0} \% \mathbf{( 7 4 2 )}$
\end{tabular}

Fuente: elaboración propia a partir de la encuesta realizada en 2018

\section{"Hasta aquí hemos llegado", la experiencia de dejar un proceso de dirección}

Un aspecto que nos interesa destacar de esta relación es que para un tesista hay un número bastante acotado de tesis que realiza a lo largo de su formación y, por tanto, también lo es el número de veces que puede resultar en un vínculo pedagógico inviable. Contrariamente, los directores pueden ocupar dicho rol en multiplicidad de ocasiones y de modo simultáneo. Esta diferencia para cada polo de la relación se observa en los resultados de nuestro estudio, ya que el $58 \%$ de los directores han experimentado, al menos una vez, la discontinuidad del vínculo pedagógico; mientras que solo el $20 \%$ de los tesistas lo han hecho 5 .

En el caso de los directores de la muestra, observamos que no hay diferencias según el género en las posibilidades de suspender un proceso de dirección de tesis. Ahora, al analizarlo en función de la edad, vemos que a medida que esta aumenta también lo hace el porcentaje de quienes han discontinuado alguna tesis. Así, entre quienes tienen 40 años o menos alrededor del $30 \%$ ha tenido esta experiencia; entre los 41 y los 49 años, un $50 \%$, y luego de los 50 años la cifra se eleva a alrededor del $60 \%$.

Semejante progresión se advierte si miramos el fenómeno a lo largo de las categorías de investigador del Conicet, las categorías más elevadas muestran los porcentajes más altos de discontinuidad en la dirección de tesis: asistente, con 33\%; adjunto, $55 \%$; independiente, con $56 \%$; principal, $70 \%$, y $52 \%$ entre los investigadores de categoría superior. Es decir, el mayor involucramiento en una de las tareas centrales del mundo académico vuelve a estos perfiles de investigadores más expuestos a la problemática.

Del mismo modo, respecto al lugar que ocupa la investigación entre las actividades diarias, quienes se dedican a la investigación como actividad

5 Es importante recordar que las encuestas tuvieron como destinatarios principales a personas que se desempeñan dentro del ámbito académico, por lo cual esta cifra puede estar afectada por dicha selección. 
principal y quienes ocupan la mitad de su tiempo en ella han vivido la discontinuidad de un proceso de dirección de tesis entre un 54\% y un 65\%. En este segundo caso es en el que se observa la mayor diferencia entre quienes han discontinuado y quienes no lo han hecho. Podría inferirse que aquí, además del alto involucramiento del director en el mundo académico, influye la competencia con otras actividades profesionales en el tiempo que se puede dedicar a la tarea de investigación (y de dirección).

Otro motivo de gran influencia es el grupo disciplinar de pertenencia del director. En la figura 1 podemos observar que en las Ciencias Sociales y Humanas se concentran los mayores porcentajes de discontinuidad de este vínculo pedagógico, al igual que es referenciado por la literatura (Bowen \& Rudenstine, 1992; De Miguel et al., 2004; Ehrenberg et al., 2010). Cabría preguntarse entonces si estos números responden a la discontinuidad en la relación director-tesista o si lo hacen al abandono de los posgrados (que puede deberse a distintos motivos). Allí observamos también que las Ciencias Biológicas y de la Salud son las que muestran menor frecuencia.

Figura 1

Distribución porcentual de los directores de tesis según la condición de haber discontinuado o no una dirección por área disciplinar

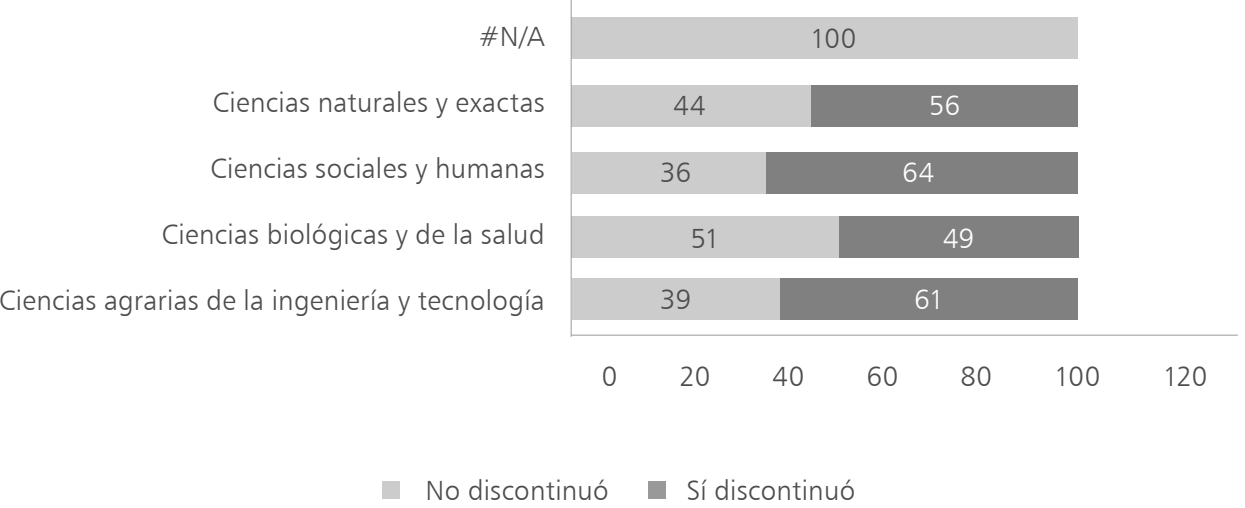

Fuente: elaboración propia a partir de la encuesta realizada en 2018

En cuanto a los tesistas encuestados, podemos inferir que, para quienes permanecen laboralmente en el sistema académico, la discontinuidad de la relación director-tesista es un fenómeno no tan frecuente. Solo el $20 \%$ ha tenido esta experiencia. Así como sucede con los directores, prácticamente no hay diferencias de género, aunque es más frecuente en mujeres (57\%) que en hombres (42\%), y para quienes no se identifican con estos géneros el porcentaje es del $1 \%$. 
Una de las preguntas más importantes para los objetivos de esta investigación es cuál es la consecuencia de esa suspensión en cuanto a la continuidad de los estudios (tabla 2). En el $65 \%$ de los casos la discontinuidad implicó un cambio de director. Solo en el $29 \%{ }^{6}$ devino en el abandono de los estudios. Estos resultados son coherentes con la conformación de la muestra, que tiene una inserción laboral mayoritaria en el ámbito científico; aunque no podemos considerarla representativa de los estudiantes de nivel de posgrado en Argentina.

En los tesistas indagamos también sobre la autoría de la decisión. En la mayoría de los casos declaran que la decisión fue propia (37\%), pero es significativo que lo sigue la categoría "no hubo una decisión" (26\%)7. Esto podría ser evidencia del escaso grado de institucionalización de la relación y de su concepción como un espacio privado (Manathunga, 2005), ya que no sería necesario declarar la cesión del proceso formativo, y del carácter implícito que puede tener la decisión de dejar de trabajar con un director o tesista. En la tabla 2 podemos ver la fuerza que toma este argumento. Cuando la decisión es explícita (ya sea compartida o por decisión de una de las partes), la consecuencia principal es el cambio de director. Contrariamente, cuando no hay una decisión, el abandono aumenta.

La ausencia de una decisión daría cuenta del paulatino abandono del trabajo en la tesis, precedido generalmente, según la literatura, por distintas actitudes de procrastinación. Según Difabio (2011), la inacción del director en estos momentos exacerba el problema. Y nuestros resultados aportan mayor evidencia sobre la deserción como consecuencia del paulatino abandono del trabajo de ambas partes de la relación pedagógica.

Tabla 2

Distribución porcentual de tesistas por consecuencia de la discontinuidad según autoría de la decisión

\begin{tabular}{|l|c|c|c|c|}
\hline & $\begin{array}{c}\text { Abandono } \\
\text { del posgrado }\end{array}$ & $\begin{array}{c}\text { Cambio } \\
\text { de director }\end{array}$ & NS/NR & Total \\
\hline La decisión fue compartida & $19 \%$ & $81 \%$ & - & $100 \%(27)$ \\
\hline La decisión fue del director & $5 \%$ & $95 \%$ & - & $100 \%(22)$ \\
\hline $\begin{array}{l}\text { No hubo una decisión explícita, simplemente } \\
\text { el tesista fue dejando de trabajar en la tesis }\end{array}$ & $56 \%$ & $34 \%$ & $10 \%$ & $100 \%(38)$ \\
\hline La decisión fue del tesista & $28 \%$ & $70 \%$ & $2 \%$ & $100 \%(54)$ \\
\hline NS/NR & & $20 \%$ & $80 \%$ & $100 \%(5)$ \\
\hline Total & $29 \%(\mathbf{4 2 )}$ & $\mathbf{6 5 \% ( 9 5 )}$ & $\mathbf{( 9 )}$ & $\mathbf{( 1 4 6 )}$ \\
\hline
\end{tabular}

Fuente: elaboración propia a partir de la encuesta realizada en 2018

6 Un $6 \%$ de los encuestados no respondieron a la pregunta.

7 Es importante resaltar que esta respuesta probablemente esté marcando una decisión de abandono de la tesis. 
En cuanto a la pertenencia disciplinar, las Ciencias Sociales y Humanas $^{8}$ son las que concentran el mayor porcentaje de discontinuidad (25\%), al igual que se indica en el caso de los directores. El resto de los grupos evidencia $15 \%$ o menos (figura 2). Como dijimos anteriormente, estas son las disciplinas reconocidas como con mayores tasas de deserción (Bowen \& Rudenstine, 1992; De Miguel et al., 2004; Ehrenberg et al., 2010).

Figura 2

Distribución porcentual de tesistas según la experiencia de haber discontinuado por área disciplinar

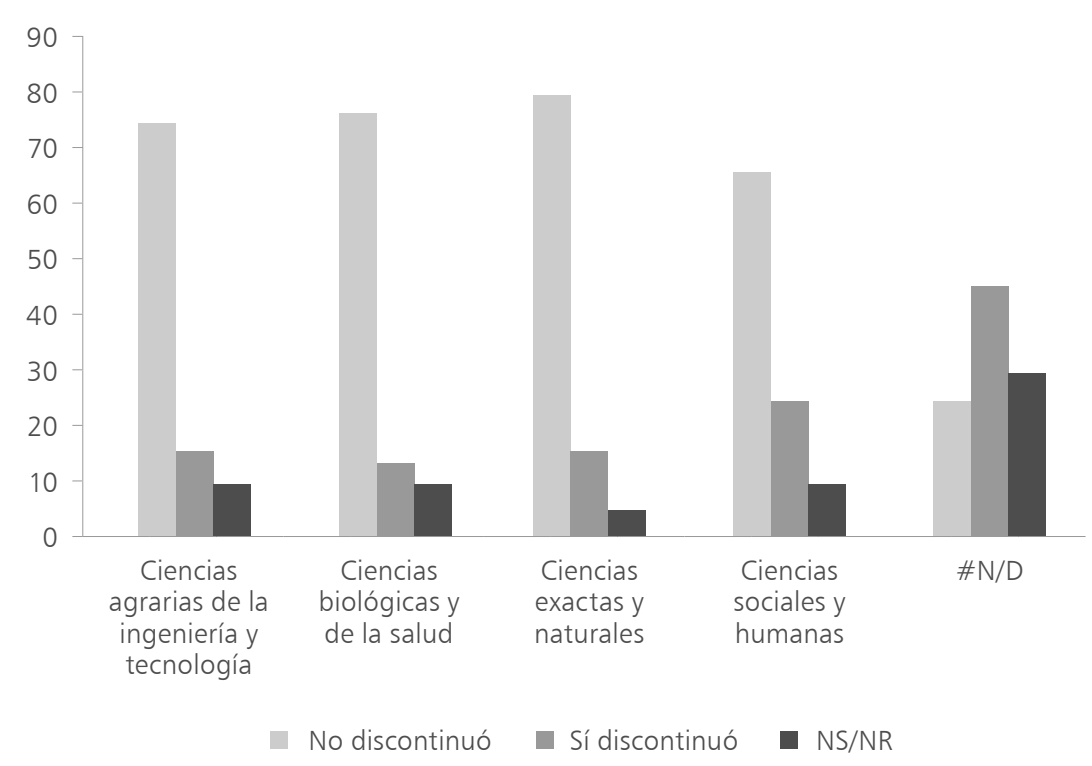

Fuente: elaboración propia a partir de la encuesta realizada en 2018

\section{Motivos que llevan a discontinuar un proceso de dirección}

Ahora bien, ipor qué director y tesista deciden disolver un proceso de dirección? Del total de directores que vivieron la discontinuidad de la relación pedagógica, el 58\% (293 encuestados) respondió la pregunta abierta que solicitaba que relatasen o ejemplificasen el motivo que los llevó a tomar la decisión. Una rápida lectura de la tabla 3 permite observar que las principales razones señaladas son ajenas a la relación pedagógica y, esencialmente, corresponden más bien al abandono del posgrado. Dado el objetivo de nuestra investigación, comenzaremos por aquellas más vinculadas con la relación pedagógica.

8 Exceptuando los casos en los que no hay datos para el área disciplinar, que son los de mayor porcentaje de discontinuidad. 
Tabla 3

Distribución porcentual de directores de tesis según los motivos que influyen en la decisión de discontinuar la relación

\begin{tabular}{l|c}
\hline Motivos de discontinuidad & Total \\
\hline Cambio vocacional/laboral & $19 \%$ \\
\hline Mal desempeño del tesista, insuficiente competencia & $16 \%$ \\
\hline Circunstancias personales del tesista (familia, mudanza, salud) & $16 \%$ \\
\hline Falta de tiempo por parte del tesista (competencia con otras actividades) & $12 \%$ \\
\hline Desaparición del tesista. El director desconoce el motivo o solo conoce sus motivos & $6 \%$ \\
\hline Discrepancias en el modo de desarrollar la investigación y/o el trabajo cotidiano & $5 \%$ \\
\hline Problemas éticos & $4 \%$ \\
\hline Problemas de vínculo con el tesista o con otros investigadores & $4 \%$ \\
\hline Cambio de tema, programa de posgrado o equipo de investigación por parte del tesista & $4 \%$ \\
\hline Inconvenientes con la beca y/o reglamentación del programa de posgrado & $4 \%$ \\
\hline Desmotivación, poco interés por la academia & $3 \%$ \\
\hline Insuficiente recepción de las indicaciones y sugerencias & $3 \%$ \\
\hline Insuficiente autonomía por parte del tesista & $2 \%$ \\
\hline Otros & $2 \%$ \\
\hline Total & $\mathbf{1 0 0 , 0 ~ ( 2 9 3 )}$ \\
\hline
\end{tabular}

Fuente: elaboración propia a partir de la encuesta realizada en 2018

El principal motivo, que se encuentra dentro del ámbito de la relación y decisión de los directores, es el mal desempeño por parte del tesista (16\%). Estas razones se han manifestado del siguiente modo:

La tesista volvió a su país de origen y no podía comprender mis instrucciones por mail, pese al tiempo que le dediqué a explicar las sugerencias y correcciones (mujer, entre 55 y 59 años, Psicología, más de seis tesis dirigidas finalizadas).

Le llevó más de un mes hacer un simple experimento de 1 día, el cual se le pidió hacer más de tres veces (otro género, entre 40 y 44 años, Biotecnología, primeras tesis dirigidas en proceso).

El tesista pretendía que el director escribiera la tesis (mujer, entre 55 y 59 años, Bioquímica, hasta seis tesis doctorales dirigidas y finalizadas).

En estas respuestas es evidente el descontento con el desempeño del tesista. Pero, si la dirección de tesis es una relación pedagógica (FernándezFastuca \& Wainerman, 2015; De la Cruz-Flores et al., 2006; Manathunga, 2007), ¿no implica esto la posibilidad del desconocimiento, la confusión, la dependencia del enseñante, propias del proceso de aprendizaje? La alianza de aprendizaje entre director y tesista se basa en la responsabilidad de 
ambos por el proceso formativo y requiere habilidad para enseñar (Halse \& Malfroy, 2010). Sin embargo, algunas de estas respuestas nos permiten ver que quienes ejercen el rol de director de tesis no siempre lo entienden como una actividad formativa. En otras, podemos ver que, ante la falta de comprensión de las indicaciones por parte del tesista, el director no logra desarrollar otras estrategias de enseñanza, por lo que se genera una discrepancia entre las necesidades de orientación del tesista y el estilo de orientación del director (Devos et al., 2016).

Otras razones manifestadas, en menor medida, por los encuestados son: las discrepancias en el modo de desarrollar la investigación (5\%), los problemas éticos (4\%) y de vinculación (4\%). Algunos de estos motivos coinciden con los resultados de Löfström \& Pyhältö (2015), al involucrar generalmente situaciones de plagio por parte del tesista y diferencias de expectativas del rol entre ambos, manifestaciones del principio "evitar el daño". Las autoras señalan que, en ocasiones, "los doctorandos tenían expectativas diferentes del rol de director, como una amistad cercana o una relación terapéutica" (Löfström \& Pyhältö, 2015, p. 2729). En palabras de los encuestados:

Tuve un caso de un estudiante de maestría que se copió en exámenes e incluso llegó a falsificar mi firma en documentación (hombre, entre 55 y 59 años, Ciencias Veterinarias, más de seis tesis de posgrado dirigidas y finalizadas).

Exigencia de atención permanente y confusión entre dirección y amistad (mujer, entre 55 y 59 años, Bioquímica, hasta seis tesis doctorales dirigidas y finalizadas).

En definitiva, todos los motivos vinculados estrechamente con la relación director-tesista parecen poner al tesista en el lugar de "estar en falta", ya sea por un desempeño insatisfactorio, por confundir los roles, por insuficiente autonomía o por cometer faltas éticas. En otras palabras, la mirada del director está siempre puesta en una concepción del tesista desde el modelo que Manathunga \& Goozée (2007) y Johnson et al. (2000) denominan "siempre listo", desconociendo, al menos parcialmente, el carácter formativo de la realización de una tesis.

Como dijimos, las principales razones son ajenas a la relación directortesista: cambio vocacional/laboral del tesista (19\%) y razones personales del tesista (16\%). En tercer lugar se encuentra la falta de tiempo por parte del tesista — "competencia" del posgrado con otras actividades- (12\%). Esto es, el $47 \%$ de los encuestados atribuye motivos por fuera del vínculo 
pedagógico. Estos datos nos permiten inferir, por un lado, que en un porcentaje importante de casos la discontinuidad de la relación es una consecuencia del abandono de los estudios y, por otro, una escasa diferenciación de estos fenómenos por parte de los directores (si consideramos que la pregunta pedía que relatasen circunstancias en las que ellos decidieron discontinuar el trabajo con el tesista). Como señala Gardner (2009), la responsabilidad por las bajas tasas de graduación en los estudios de posgrado se adjudica siempre a la deserción del estudiante, sin indagar en otras explicaciones posibles. Sin embargo, a partir de nuestros resultados, otros motivos comienzan a vislumbrarse como influyentes en la posibilidad de graduación.

En cuanto al primer punto, podríamos sostener que, en un vínculo tan próximo como es la relación director-tesista, un modo de resolver la discontinuidad consistiría en alegar razones "políticamente correctas". Esta hipótesis toma fuerza cuando profundizamos en otros motivos, como la "desaparición" del tesista y la consecuente "desorientación" del director. El primero simplemente deja de contactarse y el director no sabe por qué el tesista decidió abandonar. Por ejemplo:

Le he solicitado al tesista informe de avance y le he enviado convocatorias a eventos científicos para presentación de trabajos, pero solo responde "recibido"/"gracias". Desde que terminó el cursado de las asignaturas del postgrado y se aprobó la propuesta del plan de tesis han transcurrido más de 4 años (mujer, entre 55 y 59 años, Ciencias Geológicas, entre dos y seis tesis de posgrado dirigidas finalizadas).

En general los alumnos dejan de escribirte, algunos de ellos cambiaron el tema y el director unilateralmente [...], y otros simplemente desconozco lo que ocurrió. En algún caso reapareció luego de mucho tiempo retomando la tesis, aduciendo cuestiones familiares y laborales (hombre, entre $40 \mathrm{y}$ 45 años, Historia, hasta seis tesis en proceso).

En estos casos nuevamente podemos inferir la discrepancia entre las expectativas y las necesidades de cada uno, como señalan Devos et al. (2016). Estos autores, además, identificaron al abandono del vínculo pedagógico como una de las respuestas cuando no se pueden resolver las discrepancias. En este sentido, los casos también evidencian el carácter no institucionalizado o privado de la dirección de tesis (Manathunga, 2005).

En síntesis, respecto de los directores, es relevante señalar que en este estudio las principales razones para dejar de trabajar con un tesista se basan en el desempeño de este último, a diferencia de lo que muestra la literatura relevada (si bien esta se ha centrado principalmente en las opiniones de los tesistas, más que en las de los directores). 
En cuanto a los tesistas encuestados que vivieron la discontinuidad de la relación con el director (y no ha sido una decisión de este último), un $52 \% 9$ respondió las preguntas abiertas, lo que nos permitió profundizar en los motivos que los llevaron a tomar la decisión. Los principales motivos son (tabla 4): disconformidad con la dirección recibida (22\%), poca disponibilidad y compromiso del director (14\%) y razones personales (12\%). Con menor relevancia encontramos también: desaparición del director (8\%), cambio vocacional (8\%) y maltrato por parte del director (8\%).

Tabla 4

Distribución porcentual de tesistas según los motivos que influyen en la decisión de discontinuar la relación

\begin{tabular}{l|c}
\hline Motivos de discontinuidad & Total \\
\hline Disconformidad con la dirección recibida & $22 \%$ \\
\hline Poca disponibilidad y compromiso del director & $14 \%$ \\
\hline Razones personales & $12 \%$ \\
\hline Maltrato por parte del director & $8 \%$ \\
\hline Cambio vocacional & $8 \%$ \\
\hline Desaparición del director & $8 \%$ \\
\hline Necesidad de un director especialista en el tema & $6 \%$ \\
\hline Confusión de los objetivos y el sentido de la dirección por parte del director & $5 \%$ \\
\hline Cuestiones institucionales & $5 \%$ \\
\hline Problemas éticos con el director & $5 \%$ \\
\hline Desinterés del director por el tema de investigación del tesista & $5 \%$ \\
\hline Otros & $3 \%$ \\
\hline Demasiada exigencia del director & $2 \%$ \\
\hline Total & $\mathbf{1 0 0 \%}(\mathbf{6 5 )}$ \\
\hline
\end{tabular}

Fuente: elaboración propia a partir de la encuesta realizada en 2018

En los motivos marcados por este polo del binomio podemos advertir más incidencia de aspectos pedagógicos vinculados con la relación directortesista. En línea con nuestros resultados, Fraser \& Mathews (1999) hallaron que los tesistas apuntan a la orientación y guía crítica como las características más deseables en un director, por sobre la experiencia en el tema de investigación, resaltando también la dimensión pedagógica del vínculo director-tesista.

9 Este cálculo se ha realizado con base en los 124 encuestados que han discontinuado la relación con el director y no ha sido este último quien ha tomado la decisión de suspender el trabajo conjunto. Es decir, la decisión fue del tesista, de modo compartido o no hubo una decisión explícita. 
La disconformidad con la dirección recibida es el principal motivo señalado por los tesistas encuestados, que se expresa, por ejemplo, del siguiente modo:

Discrepancias en el "hasta donde se llega con la parte experimental". Las tesis tienen un tiempo estimado y en algún momento hay que cortar con lo experimental y escribir hasta donde se tenga. Otra cuestión es las idas y venidas con las correcciones. Corrige sobre lo ya corregido y pone trabas al avance (mujer, entre 35 y 39 años, Química, tesis doctoral).

El director cambiaba innecesariamente el tenor de sus observaciones en cada encuentro. La reescritura era incesante, eso demoraba el avance, y hacía mi trabajo imposible de responder a los requerimientos y tiempos de aplicar a becas o carrera del investigador, así como al envío de artículos para publicación. Cuando se lo hice notar, me dijo que no le importaba (mujer, entre 45 y 49 años, Letras, tesis doctoral).

En el contexto argentino, Hidalgo \& Passarella (2009) también dan cuenta de la disconformidad de los tesistas, al referir a la necesidad de mayor orientación de la que reciben por parte de sus directores. Esto lleva, por un lado, a preguntarse cuál es la función del director, cómo sería mejor orientar al tesista y cuál es el objetivo que une a ambos polos del binomio; preguntas con sentido pedagógico que la literatura ha comenzado a responder (Fernández-Fastuca, 2019; McCallin \& Nayar, 2012; MorenoBayardo, 2003; entre otros). Pero es preciso que quienes ejercen como directores también se las planteen, ya que, como manifiestan Åkerlind \& McAlpline (2017), los distintos propósitos y sentidos que se le otorgan a la tarea de dirección habilitan aprendizajes diferenciales para los tesistas. $\mathrm{O}$, como vemos en los ejemplos anteriores, imposibilitan el avance en la tesis al inhabilitar el otorgamiento de sentido a las correcciones brindadas.

Por otro lado, también se puede evidenciar desconocimiento del tesista de lo que implica realizar una investigación. En ambas respuestas parece acusarse al director de "subjetividad" a la hora de tomar decisiones sobre la investigación del tesista. Dejando de lado la posibilidad de una actitud arbitraria del director y focalizando en el carácter formativo del proceso, Delamont \& Atkinson (2001) señalan que el pasaje de la educación de grado a posgrado genera desorientación y frustración inicial en los tesistas, al descubrir que la ciencia "real" es más compleja e incierta que el mundo controlado de la formación de grado. Esas "reescrituras incesantes" pueden ser parte necesaria del proceso de producción de conocimiento original; en todo caso, la falla estaría en no explicitar adecuadamente las razones de estas. 
Por último, también la "desaparición" del director aparece como motivo que lleva a la discontinuidad de la relación pedagógica, expresada del siguiente modo por los tesistas: "Abandono del director por no responder nunca los mails" (mujer, más de 55 años, Letras, tesis doctoral); "El director se desentendió del proyecto. Literal, nunca apareció" (hombre, entre 50 y 54 años, Ciencias de la Salud, tesis doctoral).

Acorde con estos resultados tanto los tesistas como los directores tomarían actitudes de "desaparición". Entonces, cabe preguntarnos si es lícito que en un vínculo pedagógico, enmarcado en una institución de educación superior, ambas partes de la relación puedan decidir dejar de vincularse sin notificar al otro. Creemos que esto podría mostrar el escaso grado de regulación institucional que tiene la dirección de tesis, a pesar de ser una figura clave en todos los reglamentos de programas de posgrado.

Otra coincidencia que puede establecerse en los resultados de ambas partes de la relación es que entre los principales motivos que se consideran relevantes para cada polo del binomio se encuentran las carencias del opuesto. Los tesistas también posan la mirada en las faltas del director, como en el caso de su poca disponibilidad y compromiso, la disconformidad con sus habilidades para dirigir y el maltrato recibido. Esto coincide con lo hallado por Gilmore et al. (2016, p. 433), quienes señalan que los directores de tesis de su investigación "generalmente no han discutido cómo las acciones que ellos realizan, o dejan de realizar, favorecen o disminuyen la motivación de los tesistas. Además, rara vez describieron cómo sus programas o departamentos podrían apoyar las motivaciones de los tesistas". Podríamos decir que entre los encuestados todavía no hay suficiente autorreflexión sobre este vínculo pedagógico.

La confusión de los objetivos y sentido del espacio de dirección por parte del director también es uno de los motivos que refieren los tesistas. Aunque solo en unos pocos casos señalaron esta razón, lo relevante es que da cuenta de la anulación total del sentido formativo del rol de director de tesis. Por ejemplo:

El motivo principal de la desvinculación con el director de mi tesis fue que, al no coincidir con la perspectiva política de su equipo, me incitó a abandonar el lugar de trabajo y a desalentar la carrera de doctorado, al tiempo que no me convocó más a reuniones de equipo de investigación y paralizó el avance de la tesis negándose a leer mis avances. Asimismo, ejerció ciertas prácticas extorsivas de disciplinamiento asegurando que la admisión al doctorado era únicamente producto de sus mediaciones e intervenciones (otro género, entre 30 y 34 años, Filosofía, tesis doctoral). 
Es decir, en algunos casos la dirección de tesis todavía no es concebida como una relación pedagógica, sino que más bien responde a un modelo antiguo, basado en la relación maestro-discípulo, con ciertos rasgos de patronazgo.

\section{Conclusiones}

En este artículo indagamos sobre la problemática de la discontinuidad del vínculo pedagógico entre director y tesista: ¿es una "leyenda urbana" de la academia o una problemática real? Y, en el último caso, ¿cuáles son los motivos que llevan a tomar dicha decisión y las consecuencias que esta tiene? A continuación, resaltaremos las notas más relevantes sobre esta problemática que nuestros resultados nos permiten inferir.

En primer lugar, la discontinuidad de la relación director-tesista es un fenómeno que ha experimentado, al menos una vez, más de la mitad de los directores de la muestra encuestada y también algunos de los tesistas que han permanecido en el ámbito académico. Asimismo, es una problemática estrechamente relacionada con el abandono de los estudios, hecho que dificulta el análisis del fenómeno y, sobre todo, la identificación de una muestra de tesistas que se ajuste estrictamente al objeto de estudio.

En segundo lugar, la dirección de tesis es un espacio desconocido tanto por directores como tesista, de escasa reflexión sobre el rol que se ocupa en ella. Esto puede verse en que los principales motivos señalados por cada parte de la díada (dejando de lado aquellos no relacionados con nuestra pregunta de investigación) son los que focalizan en una falla o carencia del polo opuesto. Estos motivos tienen una fuerte dimensión pedagógica, que es desconocida, y se asume que "el tesista no cumple adecuadamente con las indicaciones", en lugar de pensar en el carácter procesal de toda formación. Así, parece no ser concebida como una relación pedagógica, y por ello no se adjudican motivos de este orden ni se toman decisiones explícitas sobre su fracaso. Ulteriores estudios son necesarios para profundizar en las concepciones de este vínculo para directores y tesistas.

Además, en tercer lugar, la dirección de tesis continuaría siendo un espacio privado, ajeno a la regulación institucional, que permite la "decisión" unilateral de dejarlo, que se pueda optar por simplemente "desaparecer". En este punto es importante señalar la consecuencia diferencial que tiene la decisión explícita de discontinuar la relación en comparación con la falta de ella, ya que, para el tesista, la consecuencia suele ser el abandono de los estudios. 
Dado que no se aprecian diferencias disciplinares significativas, una hipótesis que puede establecerse como resultado de esta investigación es que las altas tasas de abandono en Ciencias Sociales no se explicarían por la dirección de tesis, sino por otros factores contextuales. Serían necesarias investigaciones específicas para indagar esta hipótesis.

Este estudio tiene algunas limitaciones. Si bien se logra identificar el alcance de la problemática de la discontinuidad de la relación director-tesista, no se logra diferenciar completamente cuando el motivo es el abandono del posgrado por razones ajenas a la dirección. Además, dentro de aquellas que sí están relacionadas directamente con el vínculo pedagógico, se alcanza solo una primera identificación. Estudios ulteriores son necesarios para profundizar en ellos y analizarlos teniendo en cuenta su carácter multidimensional.

Para cerrar, podría concluirse que existen distintos niveles de reconocimiento de la dirección de tesis como tarea pedagógica. No obstante, las universidades continúan sin hacerse cargo de parte importante del proceso formativo de los tesistas. Son necesarios estudios posteriores para profundizar en ambos aspectos de la problemática. Por un lado, cuáles son las estrategias específicas que van desarrollando los directores y tesistas cuando se enfrentan con el tipo de dificultades aquí manifiestas. Por otro, un estudio que indague el rol de la universidad en la formación de posgrado y los dispositivos que pone en juego para potenciar las posibilidades de graduación de sus estudiantes.

\section{Sobre la autora}

Lorena Fernández-Fastuca es doctora en Educación de la Universidad de San Andrés y licenciada en Ciencias de la Educación de la Universidad Católica Argentina (UCA). Es becaria posdoctoral con una beca cofinanciada UCA-Conicet, y docente en UCA y Universidad de San Isidro. Su área de especialización es la pedagogía del nivel de posgrado y la dirección de tesis como práctica pedagógica.

\section{Referencias}

Abiddin, N. Z. \& West, M. (2007). Effective meeting in graduate research student supervision. Journal of Social Sciences, 3(1), 27-35. https://thescipub.com/ abstract/jssp.2007.27.35

Åkerlind, G. \& McAlpine, L. (2017). Supervising doctoral students: Variation in purpose and pedagogy. Studies in Higher Education, 42(9), 1686-1698. https://doi.org/10.1080/03075079.2015.1118031

Bartolini, A. M. (2017). La persistencia doctoral: abandono y finalización. Ciencia, Docencia y Tecnología, 28(55), 1-30. https://www.pcient.uner.edu.ar/index. php/cdyt/article/view/310/300 
Bell-Ellison, B. \& Dedrick, R. (2008). What do doctoral students value in their ideal mentor? Res High Educ, 49, 555-567. https://doi.org/10.1007/s11162008-9085-8

Bisquerra-Alzina, R. (Coord.) (2009). Metodología de la investigación educativa. La Muralla.

Bowen, W. \& Rudenstine, N. (1992). In Pursuit of the PhD. Princeton University Press.

Bradbury-Jones, C., Irving, F. \& Sambrook, S. (2007). Unity and detachment: A discourse analysis of doctoral supervision. International Journal of qualitative methods, 6(4), 81-96. https://doi.org/10.1177/160940690700600406

Carlino, P. (2005). ¿Por qué no se completan las tesis en los posgrados? Obstáculos percibidos por maestrandos en curso y magistri exitosos. Educere, 9(30), 415-420. http://erevistas.saber.ula.ve/index.php/educere/article/view/11865

Cassuto, L. (2010). Advising the struggling dissertation student. Chronicle of Higher Education, 57(17), 51-53. https://www.chronicle.com/article/Advisingthe-Struggling/125198

De la Cruz-Flores, G., García-Campos, T. \& Abreu-Hernández, L. F. (2006). Modelo integrador de la tutoría. Revista Mexicana de Investigación Educativa, 11(31), 1363-1388. http://www.scielo.org.mx/scielo.php?script=sci_ arttext\&pid=\$1405-66662006000401363\&lng=es\&tlng=

De Miguel, J., Sarabia-Heydrich, B., Vaquera, E. \& Amirah, H. (2004). ¿Sobran o faltan doctores? Emipira. Revista de Metodología de Ciencias Sociales, 7, 115-155. http://revistas.uned.es/index.php/empiria/article/view/970

Delamont, S. \& Atkinson, P. (2001). Doctoring uncertainty: Mastering craft knowledge. Social Studies of Science, 31(1), 81-107. https://doi.org/ 10.1177/030631201031001005

Delamont, S., Parry, O. \& Atkinson, P. (1998). Creating a delicate balance: The doctoral supervisor's dilemmas. Teaching in Higher Education, 3(2), 157172. https://doi.org/10.1080/1356215980030203

Deuchar, R. (2008). Facilitator, director or critical friend?: Contradiction and congruence in doctoral supervision styles. Teaching in Higher Education, 13(4), 489-500. https://doi.org/10.1080/13562510802193905

Devos, C., Boudrenghein, G., Van der Linden, N., Frebay, M., Azzi, A., Galand, B. \& Klein, O. (2016). Misfits between doctoral students and their supervisores: (How) are they regulated? International Journal of Doctoral Studies, (11), 467-486. http://www.informingscience.org/Publications/3621

Diezmann, C. (2005). Supervision and scholarly writing: Writing to learn-learning to write. Reflective Practice, 6(4), 443-457. https://doi.org/10.1080/ 14623940500300491

Difabio, H. (2011). Las funciones del tutor de la tesis doctoral en educación. Revista Mexicana de Investigación Educativa, 16(50), 935-959. http://www. scielo.org.mx/pdf/rmie/v16n50/v16n50a12.pdf

Ehrenberg, R. G., Zuckerman, H., Groen, J. A. \& Brucker, S. M. (2010). Educating Scholars. Doctoral Education in the Humanities. Princeton University Press.

Farji-Brener, A. (2007). Ser o no ser director, esa es la cuestión: reflexiones sobre cómo (no) debería ser el desarrollo de una tesis doctoral. Ecología Austral, 17, 287-292. https://www.researchgate.net/publication/262621141_Ser_o_ 
no_ser_director_esa_es_la_cuestion_reflexiones_sobre_como_no_deberia ser_el_desarrollo_de_una_tesis_doctoral

Fernández-Fastuca, L. (2019). Estilos de dirección de tesis en la formación doctoral en programas de ciencias sociales y ciencias biológicas. Espacios en Blanco, 2(29), 201-217. https://ojs2.fch.unicen.edu.ar/ojs-3.1.0/index.php/ espacios-en-blanco/article/view/357

Fernández-Fastuca, L. \& Wainerman, C. (2015) "La dirección de tesis de doctorado, ¿una práctica pedagógica? Perfiles Educativos, 37(148), 156-171. http://www.scielo.org.mx/scielo.php?script=sci_abstract\&pid=S018526982015000200010\&lng=es\&nrm=iso

Ferrer de Valero, Y. (2001). Departmental factors affecting time-to-degree and completion rates of doctoral students at one land-grant research institution. The Journal of Higher Education, 72(3), 341-367. https://doi.org/10.1080/0 0221546.2001 .11777098

Fraser, R. \& Mathews, A. (1999). An evaluation of the desirable characteristics of a supervisor. Australian Universities Review, 42(1), 5-7. https://eric. ed.gov/?id=EJ591488

Gardner, S. (2009). Student and faculty attributions of attrition in high and lowcompleting doctoral programs in the United States. Higher Education, 58, 97-112. https://doi.org/10.1007/s10734-008-9184-7

Galetto, L., Torres, C. \& Perez-Harguindeguy, N. (2007). Reflexiones sobre el desarrollo del doctorado considerando la relación orientador-orientado y la metodología pedagógica subyacente. Ecología Austral, (17), 293-298. https://www.researchgate.net/publication/262621145_Reflexiones_sobre el_desarrollo_del_doctorado_considerando_la_relacion_orientador-orien tado_y_la_metodologia_pedagogica_subyacente

Gilmore, J., Wofford, A. \& Maher, M. (2016). The flip side of the attrition coin: Faculty perceptions of factors supporting graduate student success. International Journal of Doctoral Studies, 11, 419-439. http://www.informings cience.org/Publications/3618

Halse, C. (2011). Becoming a supervisor: The impact of doctoral supervision on supervisors' learning. Studies in Higher Education, 36(5), 557-570. https:// doi.org/10.1080/03075079.2011.594593

Halse, C. \& Malfroy, J. (2010). Retheorizing supervision as professional work. Studies in Higher Education, 35(1), 79-92. https://doi.org/10.1080/0307 5070902906798

Hasrati, M. (2005). Legitimate peripheral participation and supervising Ph.D. students. Studies in Higher Education, 30(5), 557-570. https://doi. org/10.1080/03075070500249252

Hidalgo, C. \& Passarella, V. (2009). Tesistas y directores: una relación compleja e irregular. En E. Narvaja de Arnoux (Ed.), Escritura y producción de conocimiento en las carreras de posgrado (pp. 77-86). Santiago Arcos.

Jackson, P. (2015). La educación como empresa moral. En ¿Qué es la educación? (pp. 121-136). Paidós.

Johnson, L., Le, A. \& Green, B. (2000). The PhD autonomous self: Gender, rationality and postgraduate pedagogy. Studies in Higher Education, 25(2), 135-147. https://doi.org/10.1080/713696141 
Kam, B. (1997). Stlye and quality in research supervision: The supervisor dependency factor. Higher Education, 34(1), 81-103. http://www.jstor.org/ stable/3448169

Kiley, M. (2011). Developments in research supervisor training: Causes and responses. Studies in Higher Education, 36(5), 585-599. https://doi.org/10.10 80/03075079.2011.594595

Löfström, E. \& Pyhältö, K. (2015). "I don't even have time to be their friend!". Ethical dilemmas in Ph.D. supervision in the hard sciences. International Journal of Science Education, 37(16), 2721-2739. http://dx.doi.org/10.108 0/09500693.2015.1104424

Lovitts, B. (2005). Being a good course-taker is not enough: A theoretical perspective on the transition to independent research. Studies in Higher Education, 30(2), 137-154. http://citeseerx.ist.psu.edu/viewdoc/download?doi= 10.1.1.454.6298\&rep $=$ rep1\&type $=$ pdf

Mainhard, T., Van der Rijst, R. \& Van Tartwijk, J. (2009). A model for the supervisor-doctoral student relationship. High Education, 58, 359-373. https:// link.springer.com/article/10.1007/s10734-009-9199-8

Manathunga, C. (2005). The development of research supervision: Turning de light on a private space. International Journal of Academic Development, 10(1), 17-30. https://doi.org/10.1080/13601440500099977

Manathunga, C. (2007). Supervision as mentoring: The role of power and boundary crossing. Studies in Continuing Education, 29(2), 207-221. https://doi. org/10.1080/01580370701424650

Manathunga, C. \& Goozée, J. (2007). Challenging the dual assumption of the "always/already" autonomous student and effective supervisor. Teaching in Higher Education, 12(3), 309-322. https://doi.org/10.1080/ 13562510701278658

Marsh, H., Rowe, K. \& Martin, A. (2002). PhD students' evaluations of research supervision: Issues, complexities and challenges in a nationwide Australian experiment in benchmarking universities. The Journal of Higher Education, 73(3), 313-348. https://doi.org/10.1353/jhe.2002.0028

Maxwell, J. \& Miller, B. (2012). Real and virtual relationships in qualitative data analysis. En J. Maxwell, A Realistic Approach for Qualitative Research (pp. 109-125). Sage.

McCallin, A. \& Nayar, S. (2012). Postgraduate research supervision: A critical review of current practice. Teaching in Higher Education, 17(1), 63-74. https:// doi.org/10.1080/13562517.2011.590979

Moreno-Bayardo, M. G. (2003). Desde cuándo y desde dónde pensar la formación para la investigación. Educación y Ciencia, 7(28), 63-81. http://www. educacionyciencia.org/index.php/educacionyciencia/article/view/205

Murphy, N., Bain, J. \& Conrad, L. (2007). Orientations to research higher degree supervision. Higher Education, 53, 209-234. https://link.springer.com/arti cle/10.1007/s10734-005-5608-9

Subhajyoti, R. (2007). Selecting a doctoral dissertation supervisor: Analytical hierarchy approach to the multiple criteria problem. International Journal of Doctoral Studies, 2, 23-32. http://www.ijds.org/Volume2/IJDSv2p023032Ray18.pdf 
Torres-Calixto, M. (2011). La tutoría en programas de doctorado. Tensiones tutoriales doctorado en Ciencias de la Educación Rudecolombia. Revista Historia de la Educación Latinoamericana, 13(17), 315-344. http://www.scielo.org. co/pdf/rhel/v13n17/v13n17a13.pdf

Wainerman, C. (2011). Acerca de la formación de investigadores en ciencias sociales. En C. Wainerman \& R. Sautu (Eds.), La trastienda de la investigación (pp. 19-26). Manantial.

Wainerman, C. (2017). La eficiencia de doctorados en ciencias sociales y en ciencias exactas. En busca de evidencias. Debate Universitario, 6(11), 17-36. http://ppct.caicyt.gov.ar/index.php/debate-universitario/article/view/12836

Ward, A. M., \& Brennan, N. (2018). Developing a student-doctoral education fit analytical model to assess performance. Studies in Higher Education. Publish online. https://doi.org/10.1080/03075079.2018.1545758 